Journal for ImmunoTherapy of Cancer

\title{
Durable benefit from immunotherapy and accompanied lupus erythematosus in pancreatic adenocarcinoma with DNA repair deficiency
}

\author{
Xionghao Pang, ${ }^{1}$ Juanjuan Qian, ${ }^{2}$ Hua Jin, ${ }^{1}$ Lei Zhang, ${ }^{2}$ Lin Lin, ${ }^{1}$ Yuli Wang, ${ }^{3}$ Yi Lei, ${ }^{3}$ \\ Zeqiang Zhou, ${ }^{1}$ Meixiang Li, ${ }^{1}$ Henghui Zhang ${ }^{4}$
}

To cite: Pang X, Qian J, Jin $\mathrm{H}$, et al. Durable benefit from immunotherapy and accompanied lupus erythematosus in pancreatic adenocarcinoma with DNA repair deficiency. Journal for ImmunoTherapy of Cancer 2020;8:e000463. doi:10.1136/ jitc-2019-000463

- Additional material is published online only. To view please visit the journal online (http://dx.doi.org/10.1136/jitc2019-000463).

$\mathrm{XP}, \mathrm{JQ}$ and $\mathrm{HJ}$ are joint first authors.

Accepted 09 June 2020

Check for updates

(C) Author(s) (or their employer(s)) 2020. Re-use permitted under CC BY-NC. No commercial re-use. See rights and permissions. Published by BMJ.

For numbered affiliations see end of article.

Correspondence to

Dr Xionghao Pang;

nanxue168@126.com

Dr Henghui Zhang, Institute of Infectious Diseases, Beijing Ditan Hospital, Capital Medical University, Jingshundongjie8,

Beijing, China;

zhhbao@ccmu.edu.cn

\section{ABSTRACT}

Background Clinical trials showed limited benefit of anti-PD-1 (programmed cell death 1) monotherapy in pancreatic adenocarcinoma patients and immune-related adverse events caused by immune checkpoint inhibitors were rarely reported in pancreatic adenocarcinoma. Here, we report the first case of durable benefit along with systemic lupus erythematosus following immunotherapy in mismatch repair-proficient pancreatic cancer.

Case presentation We describe a 57-year-old woman with resected stage IIIB pancreatic cancer who underwent several lines of conventional chemotherapy after multiple lymph node metastases. When the disease progressed again, the patient received an off-label treatment with pembrolizumab (100 mg every 3 weeks). After four cycles of immunotherapy treatment, CA19-9 level rapidly decreased to normal and the lymph node metastases reduced dramatically in volume, demonstrating a partial response to the therapy by RECIST 1.1 criteria. She continued on pembrolizumab and a total of eight cycles of administration she had received. Her lesions showed consistent reduction in size even when the medication had been stopped. Actually the patient experienced durable benefit from anti-PD-1 therapy for more than 4 years and she is still in good condition without tumor relapses to date. Besides, she was diagnosed with systemic lupus erythematosus 2 months after the last dose of pembrolizumab. Molecular profiling identified two deleterious $P A L B 2$ alterations including a germline mutation (PALB2 c.3114-1G $>$ A) and a somatic mutation (PALB2 c. $2514+1 G>C$ ) in this patient, suggesting the potential of DNA homologous recombination deficiency. Multiplex immunohistochemistry and RNA-seq results revealed a brisk immune cell infiltration in her resected primary lesion. Additionally, humanleukocyte antigen (HLA) typing assay identified two previously reported systemic lupus erythematosus risk alleles HLA-DRB1*15:01 and HLA-DQB1 ${ }^{*} 06: 02$ in this patient.

Conclusions The deleterious mutations of PALB2 closely related to homologous recombination deficiency or alterations of DNA damage response and repair genes might be promising biomarkers for predicting efficacy of immune checkpoint inhibitors in pancreatic adenocarcinoma. Genetic correlation behind immunotherapy-induced systemic lupus erythematosus and associated mechanism remain to be elucidated.

\section{INTRODUCTION}

Pancreatic adenocarcinoma (PAAD) is one of the most refractory malignancies with poor prognosis after radical surgery. In recent years, immune checkpoint blockade-based immunotherapies have gained encouraging success in multiple solid tumors, however, single-agent immune checkpoint inhibitors (CPIs) fail to elicit efficacy in PAAD patients except pembrolizumab in certain individuals with mismatch repair deficiency. ${ }^{12}$ There is little known about whether there are other biomarkers for predicting response to CPI treatment in PAAD. It should be noted that $3.9 \%$ to $9.7 \%$ of pancreatic ductal adenocarcinoma individuals carry pathogenic/likely pathogenic germline alterations, especially those alterations involved in homologous recombination deficiency (HRD) or DNA damage response and repair (DDR) genes including BRCA1/2, PALB2, ATM, etc. ${ }^{34}$ Moreover, the deleterious DDR gene variants have revealed the potential of predicting responsiveness to anti-PD-1 (programmed cell death 1)/PD-L1 (programmed death-ligand 1) therapy in bladder urothelial carcinoma ${ }^{5}$ and non-small cell lung cancer patients, ${ }^{6}$ whereas there are few reports of survival benefit in PAAD patients receiving CPIs.

As the increasing prevalence of CPI treatment in solid tumors, the special feature of side effects, that is, the immune related adverse events (irAEs), have gained wide attention. The diverse irAEs bring challenges in management of cancer patients during immunotherapy treatment. On the other hand, studies have found that certain irAEs are associated with improved response and prognosis in cancer patients treated with CPIs. ${ }^{78}$ In general, it is vital to explore the underlying mechanisms of irAEs, especially genetic factors. ${ }^{9}$ 
A

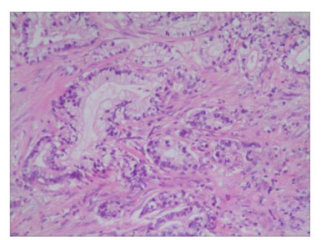

B

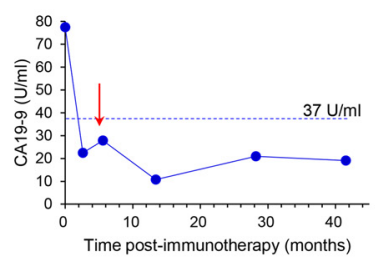

C
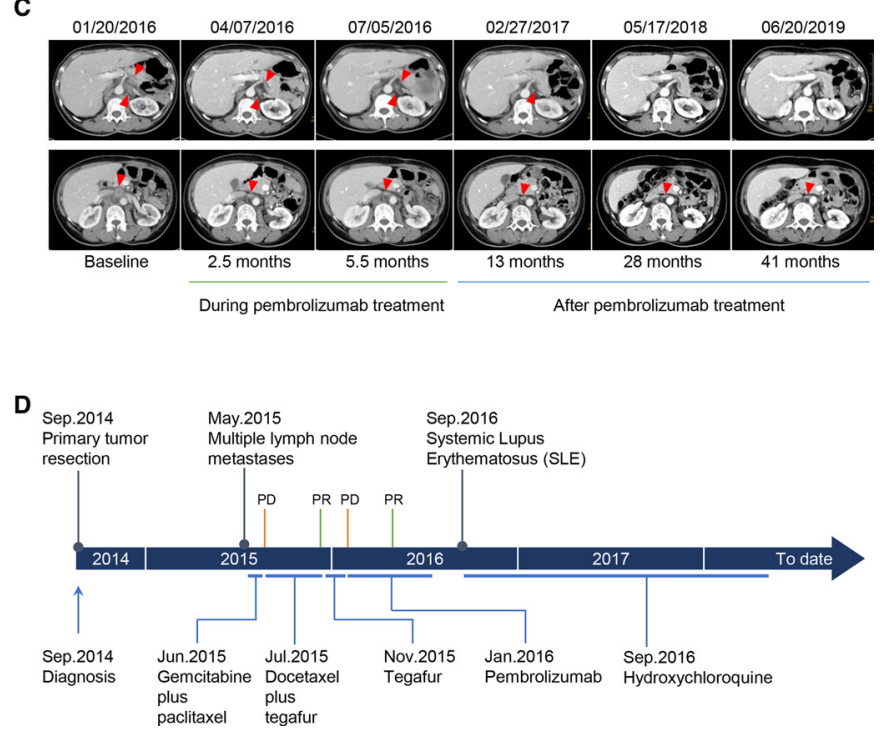

Figure 1 Clinical course of the patient. (A) Histological characteristic of the moderately differentiated adenocarcinoma of the pancreas (hematoxylin-eosin stain; original magnification $\times 400)$. (B) Serum CA19-9 levels during and after treatment with pembrolizumab (red arrow indicates the last dose of pembrolizumab). (C) Representative contrastenhanced CT images of the abdomen in venous phase, showing patient's recurrent lymph node lesions during and after treatment with pembrolizumab. A 2.5-month follow-up scan demonstrated partial response of the disease. (the top row: retroperitoneal lymph nodes; the bottom row: abdominal para-aortic lymph node; lesions are marked by red triangle). (D) Time line of clinical events. PD, progressive disease; PR, partial response.

Here we report an exceptional case of recurrent PAAD individual with microsatellite stability (MSS)/proficient mismatch repair who experienced durable survival benefit (more than 4years) along with systemic lupus erythematosus (SLE) after receiving pembrolizumab. Two deleterious $P A L B 2$ mutations including a germline mutation in combination with several humanleukocyte antigen (HLA) class II risk loci for SLE identified in this patient may account for her outcomes under CPI treatment.

\section{CASE PRESENTATION}

A 57-year-old Chinese woman was diagnosed with a stage IIIB moderately-differentiated pancreatic adenocarcinoma (figure 1A) and underwent distal pancreatectomy and en-bloc splenectomy in September 2014. Eight months later, a follow-up contrast-enhanced CT scan revealed enlargement of her multiple abdominal lymph nodes. Then the patient was treated with two cycles of chemotherapy with gemcitabine $(1200 \mathrm{mg})$ plus paclitaxel liposome $(180 \mathrm{mg})$ on days 1 and 8 , every 3 weeks. However, the re-examination of CT demonstrated progression of disease (PD) according to Response Evaluation Criteria in Solid Tumors (RECIST, V.1.1). Next, she started receiving docetaxel $(110 \mathrm{mg})$ intravenously on days 1 , plus tegafur (50 mg, two times per day) orally on days 1 to 14 , every 3 weeks. CT scan showed a partial response (PR) following six cycles of the chemotherapy. In November 2015, docetaxel was discontinued and tegafur regimen was used for maintenance chemotherapy. After two cycles of tegafur monotherapy, a follow-up CT scan showed the PD again. CT imaging revealed the presence of retroperitoneal lymph node metastases with two nodes of $2.4 \times 2.1 \mathrm{~cm}$ and $2.9 \times 1.7 \mathrm{~cm}$, respectively, as well as abdominal para-aortic lymph node metastasis with a node of $2.4 \times 2.2 \mathrm{~cm}$. Whereafter, this patient decided to pursue an off-label use of pembrolizumab $(100 \mathrm{mg}$, every 3 weeks) on January 21, 2016. Fortunately, radiological results demonstrated a PR after four cycles of immunotherapy. Moreover, her serum level of CA19-9 rapidly dropped down to normal (below $37 \mathrm{U} / \mathrm{mL}$ ) (figure 1B). Next, she continued to receive another four cycles of pembrolizumab with the same dosage and administration as before and durable benefit was observed (figure 1C). Notably, after her last cycle of pembrolizumab treatment in July 2016, the adverse effects started to appear, characterized by allergic dermatitis, fever, nausea and vomiting. In September 2016, she was diagnosed with SLE based on her clinical features and autoantibody in serum, positive antinuclear antibody of 1:320 titer (speckled patterns) and antibodies to Sjögren's-syndrome-related antigen A (SS-A). Subsequently, she was administrated with hydroxychloroquine ( $200 \mathrm{mg}$, daily) to control SLE until March 2019. In the meantime, her recurrent tumor lesions of retroperitoneal nodes achieved near complete remission and the para-aortic nodes maintained a reduced size. This patient has neither history of autoimmune disease nor family history of cancer. Her whole disease course and clinical outcomes were summarized in figure 1D. From initiation of the immunotherapy to date, the patient experienced durable survival benefit of more than 4 years, and she had a good performance status when this case report was revised.

\section{METHODS}

To identify the involvement of genomic features as well as tumor immune microenvironment signature in this unexpected response to immune checkpoint blockade and underlying genetic association with pembrolizumabinduced systemic lupus erythematosus. We performed molecular analyzes of the patient's resected primary tumor tissue, paracancerous tissue and peripheral blood sample in a College of American Pathologists accredited laboratory (GeneCast Biotechnology Co, Beijing) with consent of the patient. 
Tumor immune microenvironment was examined by multiplex immunohistochemistry (mIHC) and RNA sequencing. Two resected tumor tissue sections were used for mIHC staining: one for anti-CD3 (clone LN10), antiCD8 (clone SP16), anti-PD-1 (clone UMAB199); the other for anti-CD4 (clone UMAB64), anti-CD68 (clone KP1), anti-CD163 (clone 10D6); all the primary antibodies were from ZSGB-BIO, Beijing, China. The stained slides were scanned using the PerkinElmer Vectra and analyzed by inForm Advanced Image Analysis software (PerkinElmer, Massachusetts, USA). Positive cells were defined as cells with true immunofluorescence signal detected and with right expression pattern. More than 12 fields per slide were selected to calculate the number, percentage and density of positive cells under the $200 \times$ magnification. Mean percentage of specific positive cells in all nucleated cells of the tumor stroma from the selected fields was used for analyzes. RNA sequencing based gene expression profiling was performed on resected tumor tissue and paracancerous tissue by the Oncomine Immune Response Research Assay (Thermo Fisher Scientific, USA). Gene expression levels of approximately 400 genes relevant to oncology and immunology were analyzed. Five immune signatures were defined to represent $\mathrm{T}$ cell-inflamed features, including $\mathrm{T}$ cell markers $(\mathrm{CD} 2$, CD3D, CD3E, CD4, CD40LG, CD8A and CD8B), cytolytic markers (GNLY, GZMA, GZMB and PRF1), IFN- $\gamma$ signature (CXCL10, CXCL9, HLA-DRA, IDO1, IFNG and STAT1), HLA molecules and chemokines. The signature scores were calculated by averaging the $\log 10$ transformed expression value of the involved genes.

Genetic analyzes were used to explore the mechanism of immunotherapy response and the induced autoimmune disease. HLA genotyping of the peripheral blood lymphocytes was performed via PCR amplification with sequence based typing methodology and DNA sequencing analysis of resected tumor and peripheral blood sample was performed using next generation sequencing of a customized gene panel, including 543 cancer related genes.

PD-L1 expression and mismatch repair protein expression were also examined with regular methods. For more details of the experimental methods, please see the online additional file 1 . No statistical test was performed as there is only one patient and we did not investigate the differences between samples.

\section{RESULTS}

Targeted sequencing results show a common driver mutation $K R A S$ p.G12V $(8.72 \%)$ along with two splicing mutations of DDR gene PALB2 in her resected primary lesion (online supplementary table S1). No alterations of other major driver genes (ie, TP53, CDKN2A, SMAD4) in PAAD were identified. Of note, both of the two PALB2 mutations are viewed as likely pathogenic based on ClinVar database, suggesting the probable deficiency of DNA homologous recombination. Among them, PALB2 c.3114-1G >A

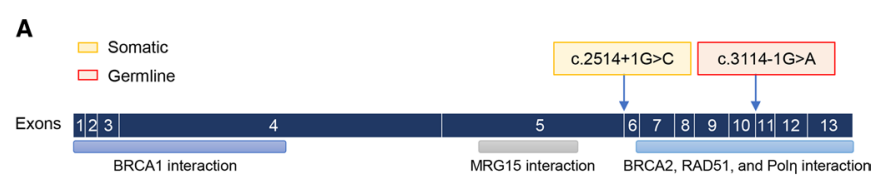

B

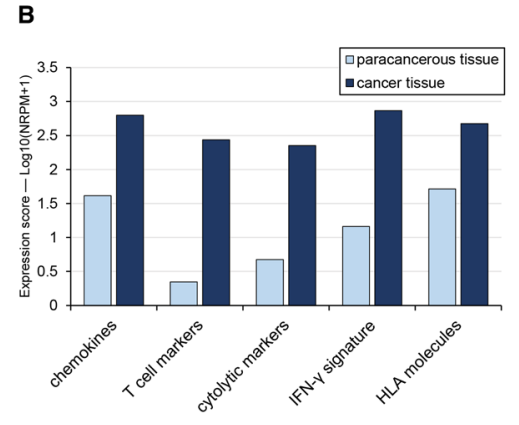

D

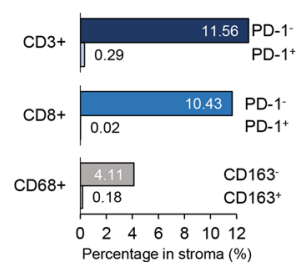

E

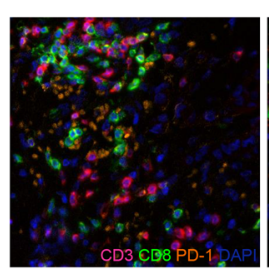

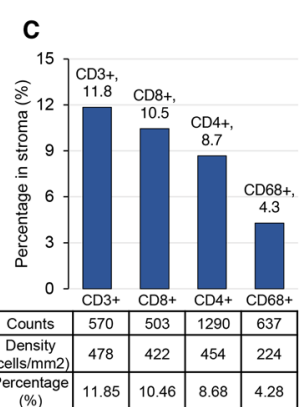

F
Figure 2 PALB2 mutations and immunologic characteristics of the patient. (A) Schematic representation of PALB2 gene with the two splicing mutations indicated. (B) Immune response signature scores of primary tumor and matched paracancerous tissue. (C) Quantitative distribution of tumor infiltrated T cell and macrophage in the stroma of the primary tumor. (D) Distribution of PD-1 expression on CD3 or CD8 positive T cells and CD163 expression on $\mathrm{CD} 68$ positive macrophages in stroma. ( $E$ and $F$ ) Representative histological images of the resected tumor stained by multiplex immunofluorescence, showing the brisk immune cell infiltration in tumor microenvironment. Original magnification $\times 200$. HLA, human leukocyte antigen; PD-1, programmed cell death 1 .

was a germline mutation simultaneously detected in her peripheral blood cells, while PALB2 c.2514+1G $>\mathrm{C}$ $(8.32 \%)$ was a somatic mutation (figure $2 \mathrm{~A}$ ).

Tumor immune microenvironment signature of this patient was examined by expression profiling of 395 immune related genes and analysis of immune cell subsets in situ by mIHC. Compared with paracancerous tissue, higher expression levels of cytolytic markers, $\mathrm{T}$ cell markers, IFN- $\gamma$ signature, chemokines as well as HLA molecules were observed in cancer tissue, demonstrating a T cell-inflamed tumor microenvironment (figure 2B). Global expression levels of 35 pre-set gene function signatures were also evaluated and immune signature scores of 21 functional pathways are at least 1.5-fold differentially changed in cancer tissue relative to paracancerous tissue, most of which are favorable for antitumor immune (online supplementary figure S1). In consistent with the expression profiling results, there were relatively high infiltration degrees of CD3 or CD8 positive $\mathrm{T}$ cells in stroma of cancer specimen examined by multiplex immunohistochemistry (figure 2C-F). Cytotoxic T cells (CD8+) 
Table 1 Human leukocyte antigen alleles of the patient

\begin{tabular}{|c|c|c|c|}
\hline HLA loci & Allele & $\begin{array}{l}\text { Association } \\
\text { with SLE } \\
\text { susceptibility }\end{array}$ & $\begin{array}{l}\text { Population } \\
\text { (reference) }\end{array}$ \\
\hline \multirow[t]{2}{*}{ HLA-A } & $A^{*} 02: 01$ & - & NA \\
\hline & $A^{* 11: 01}$ & Uncertain & $\begin{array}{l}\text { Malays and } \\
\text { Chinese }^{18}\end{array}$ \\
\hline \multirow[t]{2}{*}{ HLA-B } & $B^{*} 15: 01$ & - & NA \\
\hline & $B^{\star} 54: 01$ & - & NA \\
\hline \multirow[t]{2}{*}{ HLA-C } & $C^{\star} 03: 03$ & - & NA \\
\hline & $C^{\star} 01: 02$ & - & NA \\
\hline \multirow[t]{2}{*}{ HLA-DQ } & DQB1*05:01 & Risk factor & $\begin{array}{l}\text { Chinese } \\
\text { Taiwan }^{19}\end{array}$ \\
\hline & DQB1*06:02 & Risk factor & $\begin{array}{l}\text { Eastern } \\
\text { Asians }^{11} \\
\text { European }^{1020} \\
\text { Saudis }^{21}\end{array}$ \\
\hline \multirow[t]{2}{*}{ HLA-DR } & DRB1*01:01 & - & NA \\
\hline & DRB1*15:01 & Risk factor & $\begin{array}{l}\text { Eastern } \\
\text { Asians }^{11} \\
\text { Chinese } \\
\text { Taiwan }^{1922} \\
\text { Japanese }^{23} \\
\text { Koreans }^{24} \\
\text { European }^{1020}\end{array}$ \\
\hline \multirow[t]{2}{*}{ HLA-DP } & DPB1*04:02 & - & NA \\
\hline & DPB1*05:01 & Uncertain & Japanese 25 \\
\hline
\end{tabular}

Alleles in bold font are considered to be more likely to increase the risk of SLE.

-, unknown; HLA, human leukocyte antigen; NA, not available;

SLE, systemic lupus erythematosus.

and helper T cells (CD4+) account for about $10 \%$ of all cells in tumor stroma, respectively, and macrophages (CD68+) has a proportion of 4\% (figure 2C). Moreover, exhausted or inhibitory cells such as PD-1 positive CD3 or $\mathrm{CD} 8 \mathrm{~T}$ cells or $\mathrm{CD} 68^{+} \mathrm{CD} 163^{+}$cells were also explored, each accounts for tiny proportion in corresponding immune cell subset (figure 2D; online supplementary figure S2), indicating good activity and antitumor potential of the tumor infiltrating lymphocytes or macrophages. Collectively, primary tumor of this pancreatic patient has a favorable baseline immune microenvironment.

Additionally, PD-L1 (SP142 staining) was absent and four mismatch repair proteins (MLH1, MSH2, MSH6 and PSM2) were proficient in this patient's tumor (online supplementary figure S3). Likewise, her microsatellite instability status was MSS (online supplementary figure S4). Given the close relationship of HLA alleles with the susceptibility to SLE, ${ }^{10}$ we performed HLA genotyping of normal DNA from her peripheral blood cells. Not surprisingly, two SLE risk alleles HLA-DRB1*15:01 and HLADQB $1 * 06: 02$ previously reported in the Eastern Asians ${ }^{11}$ were identified. Besides, there were some other HLA alleles potentially related to SLE in this patient (table 1).

\section{DISCUSSION AND CONCLUSIONS}

The pancreatic ductal adenocarcinoma patients with HRD have shown association with enhanced expression of antitumor immunity related molecules and active immune status in previous research, ${ }^{12}$ which is in agreement with our findings of this pancreatic cancer patient harboring deleterious PALB2 mutations. There were obviously higher levels of cytolytic markers in her resected cancer tissue than in paracancerous tissue as well as more infiltrates of $\mathrm{CD}^{+} \mathrm{T}$ cells in tumor stroma. Correspondingly, she responded well to CPI treatment with pembrolizumab and achieved more than 4-year survival benefit. It should be noted that this individual had paired deleterious mutations of $P A L B 2$, each one for germline and somatic, while about half of PAAD patients arising from pathogenic germline alterations of homologous recombination (HR) genes lack somatic variants of HR genes ('second hit') in their tumors and these patients may not be true HRD. ${ }^{13}$ Theoretically, co-occurrence of germline and somatic HR gene alterations might bring about higher number of tumor-specific neoantigens along with relatively activated immune environment. ${ }^{12}$ Given this, we speculated that this patient's paired PALB2 mutations probably contributed to her exceptional good outcomes after pembrolizumab treatment, although to our knowledge there are hardly any clinical studies reporting HR gene variants as predictive biomarkers for CPI monotherapy in PAAD patients to date.

During cancer immunotherapy, irAEs consisting of rash, colitis, pneumonitis, and so on, are not uncommon as the increasing clinical use of CPIs. ${ }^{14}$ Nonetheless, secondary lupus erythematosus following CPI treatment is fairly rare. In terms of mechanism, two aforementioned known HLA class II (HLA-II) germline alleles susceptible to SLE in combination with the exposure to pembrolizumab may jointly lead to the unusual adverse event of this patient. The two SLE risk alleles HLA-DRB1*15:01 and HLADQB1*06:02 might confer a high level of immunity to this patient or even put her in a pre-autoimmunity state. As an external factor, CPI could just trigger the development of SLE. Interestingly, a substantial association between the development of irAEs and survival benefit from antiPD-1/PD-L1 antibodies has been recently identified in 1747 patients. ${ }^{8}$ In addition, it was previously reported that among five efficacy evaluable cancer patients with the occurrence of lupus erythematosus after receiving CPIs, three individuals had complete or partial remission and two of them were treated with pembrolizumab. ${ }^{15}$ It is impressive that CPI showed remarkable efficacy in this patient with HRD pancreatic cancer carrying two SLE risk alleles, which may inspire future investigation of the intrinsic correlation between efficacy and side effects of checkpoint inhibitors in tumors. Predictive biomarkers of immunotherapy-related toxicity are worth studying, like autoantibodies ${ }^{16}$ or genetic factors. ${ }^{9}$

Notably, the duration of exposure to pembrolizumab in this PAAD patient was just 6 months $(100 \mathrm{mg}$, every three weeks). However, she gained lasting survival benefit along 
with lupus erythematosus until now. In consideration of the potential role of HLA-II molecules in immunotherapy, ${ }^{17}$ it is plausible that her specific HLA-II genotype perhaps not only involves in irAEs but also is beneficial for CPI treatment.

In summary, DNA repair deficiency characterized by deleterious germline and somatic alterations of $P A L B 2$ as well as certain HLA-II germline alleles related to lupus erythematosus may be promising biomarkers for antiPD-1/PD-L1 therapy in PAAD patients.

\section{Author affiliations}

${ }^{1}$ Department of Oncology, The First Affiliated Hospital of Shenzhen University, Health Science Center, Shenzhen Second People's Hospital, Shenzhen, China

${ }^{2}$ Genecast Precision Medicine Technology Institute, Beijing, China

${ }^{3}$ Department of Radiology, The First Affiliated Hospital of Shenzhen University, Health Science Center, Shenzhen Second People's Hospital, Shenzhen, China ${ }^{4}$ Institute of Infectious Diseases, Beijing Ditan Hospital, Capital Medical University, Beijing, China

Acknowledgements The authors wish to gratefully acknowledge the patient and her family for allowing us to publish her clinical case.

Contributors XP, JQ and $\mathrm{HJ}$ contributed equally to this work and are co-first authors. XP and JQ had full access to all of the data in the study and take responsibility for the integrity of the data and the accuracy of the data analyzes. XP, JQ and HJ designed the study. LL, ZZ, and ML collected the data. YW and YL performed radiological data analysis and provided the imaging figures. JQ, HJ, LL, $\mathrm{ZZ}$ and $\mathrm{ML}$ analyzed the data. JQ drafted the manuscript. XP was the main treating physician. LZ, XP and HZ contributed to the interpretation of the results and critical revision of the manuscript for important intellectual content. All authors have read and approved the final version manuscript. XP and $\mathrm{HZ}$ are the study guarantors.

Funding This work was supported by grants from the National Key Sci-Tech Special Project of China (No. 2018ZX10302207).

Competing interests None declared.

Patient consent for publication Obtained.

Ethics approval This study was approved by the Research Ethics Committee of Shenzhen Second People's Hospital (Shenzhen, China), and the patient gave informed written consent.

Provenance and peer review Not commissioned; externally peer reviewed.

Open access This is an open access article distributed in accordance with the Creative Commons Attribution Non Commercial (CC BY-NC 4.0) license, which permits others to distribute, remix, adapt, build upon this work non-commercially, and license their derivative works on different terms, provided the original work is properly cited, appropriate credit is given, any changes made indicated, and the use is non-commercial. See http://creativecommons.org/licenses/by-nc/4.0/.

\section{REFERENCES}

1 Henriksen A, Dyhl-Polk A, Chen I, et al. Checkpoint inhibitors in pancreatic cancer. Cancer Treat Rev 2019;78:17-30.

2 Le DT, Durham JN, Smith KN, et al. Mismatch repair deficiency predicts response of solid tumors to PD-1 blockade. Science 2017;357:409-13.

3 Shindo K, Yu J, Suenaga M, et al. Deleterious germline mutations in patients with apparently sporadic pancreatic adenocarcinoma. J Clin Oncol 2017;35:3382-90.

4 Yurgelun MB, Chittenden AB, Morales-Oyarvide V, et al. Germline cancer susceptibility gene variants, somatic second hits, and survival outcomes in patients with resected pancreatic cancer. Genet Med 2019;21:213-23.

5 Teo MY, Seier K, Ostrovnaya I, et al. Alterations in DNA damage response and repair genes as potential marker of clinical benefit from PD-1/PD-L1 blockade in advanced urothelial cancers. J Clin Oncol 2018;36:1685-94.

6 Ricciuti B, Cheng ML, Recondo G, et al. DNA damage response gene alterations are associated with high tumor mutational burden and clinical benefit from programmed death 1 axis inhibition in non-small cell lung cancer. J Clin Oncol 2019;37:9077.

7 Lo JA, Fisher DE, Flaherty KT. Prognostic significance of cutaneous adverse events associated with pembrolizumab therapy. JAMA Oncol 2015;1:1340-1.

8 Maher VE, Fernandes LL, Weinstock C, et al. Analysis of the association between adverse events and outcome in patients receiving a programmed death protein 1 or programmed death ligand 1 antibody. J Clin Oncol 2019;37:2730-7.

9 Khan Z, Hammer C, Guardino E, et al. Mechanisms of immunerelated adverse events associated with immune checkpoint blockade: using germline genetics to develop a personalized approach. Genome Med 2019;11:39.

10 Teruel M, Alarcón-Riquelme ME. The genetic basis of systemic lupus erythematosus: what are the risk factors and what have we learned. $J$ Autoimmun 2016;74:161-75.

11 Molineros JE, Looger LL, Kim K, et al. Amino acid signatures of HLA class-I and II molecules are strongly associated with SLE susceptibility and autoantibody production in eastern Asians. PLOS Genet 2019;15:e1008092.

12 Connor AA, Denroche RE, Jang GH, et al. Association of distinct mutational signatures with correlates of increased immune activity in pancreatic ductal adenocarcinoma. JAMA Oncol 2017;3:774-83.

13 Wood LD, Yurgelun MB, Goggins MG. Genetics of familial and sporadic pancreatic cancer. Gastroenterology 2019;156:2041-55.

14 Thompson JA, Schneider BJ, Brahmer J, et al. Management of Immunotherapy-Related toxicities, version 1.2019. J Nat/ Compr Canc Netw 2019;17:255-89.

15 Michot J-M, Fusellier M, Champiat S, et al. Drug-induced lupus erythematosus following immunotherapy with anti-programmed death-(ligand) 1. Ann Rheum Dis 2019;78:e67.

16 de Moel EC, Rozeman EA, Kapiteijn EH, et al. Autoantibody development under treatment with Immune-Checkpoint inhibitors. Cancer Immunol Res 2019;7:6-11.

17 Alspach E, Lussier DM, Miceli AP, et al. MHC-II neoantigens shape tumour immunity and response to immunotherapy. Nature 2019;574:696-701.

18 Mohd-Yusuf Y, Phipps ME, Chow SK, et al. HLA-A*11 and novel associations in Malays and Chinese with systemic lupus erythematosus. Immunol Lett 2011;139:68-72.

19 Lu LY, Ding WZ, Fici D, et al. Molecular analysis of major histocompatibility complex allelic associations with systemic lupus erythematosus in Taiwan. Arthritis Rheum 1997;40:1138-45.

20 Graham RR, Ortmann W, Rodine P, et al. Specific combinations of HLA-DR2 and DR3 class II haplotypes contribute graded risk for disease susceptibility and autoantibodies in human SLE. Eur J Hum Genet 2007:15:823-30.

21 Al-Motwee S, Jawdat D, Jehani GS, et al. Association of HLADRB $1^{*} 15$ and HLADQB $1^{*} 06$ with SLE in Saudis. Ann Saudi Med 2013;33:229-34.

22 Pan CF, Wu CJ, Chen HH, et al. Molecular analysis of HLA-DRB1 allelic associations with systemic lupus erythematous and lupus nephritis in Taiwan. Lupus 2009;18:698-704.

23 Shimane K, Kochi Y, Suzuki A, et al. An association analysis of HLA-DRB1 with systemic lupus erythematosus and rheumatoid arthritis in a Japanese population: effects of *09:01 allele on disease phenotypes. Rheumatology 2013;52:1172-82.

24 Bang S-Y, Choi J-Y, Park S, et al. Brief report: influence of HLA-DRB1 susceptibility alleles on the clinical subphenotypes of systemic lupus erythematosus in Koreans. Arthritis Rheumatol 2016;68:1190-6.

25 Furukawa H, Oka S, Shimada K, et al. Association of increased frequencies of HLA-DPB $1{ }^{*}$ 05:01 with the presence of anti-Ro/SS-A and anti-La/SS-B antibodies in Japanese rheumatoid arthritis and systemic lupus erythematosus patients. PLoS One 2013;8:e53910. 